\title{
Stage IC Eyelid Carcinoma AJCC v7
}

National Cancer Institute

\section{Source}

National Cancer Institute. Stage IC Eyelid Carcinoma AICC V7. NCI Thesaurus. Code C88125.

Stage IC includes: T2b, N0, M0. T2b: Tumor more than $10 \mathrm{~mm}$, but not more than 20 $\mathrm{mm}$ in greatest dimension. Or, tumor involves full thickness of the eyelid. NO: No regional lymph node metastasis. M0: No distant metastasis. (AJCC 7th ed.) 